Commissioned response to Filipe O. Costa \& Gary R. Carvalho, 'The Barcode of Life Initiative: Synopsis and Prospective Societal Impacts of DNA Barcoding of Fish'

\title{
Real but modest gains from genetic barcoding
}

\section{JOHN DUPRÉ ${ }^{1}$}

Costa and Carvalho ${ }^{2}$ make a compelling case for the practical utility of barcoding fish. Essentially the barcode, the precise sequence of a carefully chosen few hundred base pairs of a mitochondrial gene found in all eukaryotes, is intended as a definitive taxonomic criterion that can be added to the existing description of a species, but that has the enormous advantage of being applicable to any part of the organism. No existing part of most taxonomic descriptions can be applied to a fish finger, remains of animals in a fish's stomach or, probably, a detached shark's fin. Since, as they explain, there are important practical contexts in which it is desirable to relate such objects to their species of origin, detecting fraudulent fishmongers or violations of fishery preservation law, for example, the potential benefits are clear. They might also, in imaginable future circumstances, come to be of considerable benefit in providing definitive classifications for field biologists without easy access to relevant kinds of taxonomic expertise.

It is much more difficult to understand how the introduction of this technique will revolutionize the practice of taxonomy or enable the 'completion of the biodiversity catalogue within the reach of a single generation'. I'll leave aside for a moment the fact that this project is explicitly limited to eukaryotes (and in practice has only so far been applied with much success to animals), and therefore that this hypothetical catalogue will be missing out the very large majority of organisms and probably the majority of kinds of organisms. My first point is merely that the limiting factor in cataloguing life will surely continue to be the number of properly trained taxonomists.

Perhaps the most important theoretical point is that the introduction of genetic barcodes does nothing to solve the traditional problem of determining what a species is. A few decades ago, partly due to the effective advocacy of Ernst Mayr, it was widely believed (if by no means universally by professional systematists) that species could be defined as reproductively isolated groups - the so-called Biological Species Concept. Unfortunately it became increasingly clear that reproductive isolation was neither a necessary nor a sufficient condition for maintenance of the morphologically stable kinds generally agreed to be species. That reproductive isolation was not necessary was classically illustrated by the case of oaks, ${ }^{3}$ in which distinct species appeared to have existed for long periods of time despite continuous and substantial interbreeding, but it now appears that many other groups of organisms might have been chosen to make the point. Lack of sufficiency was demonstrated by the existence of species dispersed among isolated populations, physically unable to interact and mate, yet showing no significant divergence. ${ }^{4}$ 
The biological species concept assumed a picture of evolution as consisting of a branching tree in which the initiation of a branch could be defined by reference to the establishment of reproductive isolation between the organisms represented by the new and the originating branches. If a group of organisms conforms to this model, and a reasonable period of time has passed since the occurrence of the speciation event marked by the branch in the tree, an appropriate mitochondrial gene sequence is likely to provide a good criterion for species membership. ${ }^{5}$ However, a likely explanation for the problems with the biological species concept is that local diversity within a genus or even higher taxon is maintained by ecological differentiation rather than reproductive isolation. A compelling reason for believing this is the fact that interspecific hybridization is proving to be far more common than had for a long time been thought, even in groups such as birds, which have been widely taken to be a paradigm for application of the biological species concept. ${ }^{6}$ Hybridization involves, by definition, exchange of genetic material, and hence makes the use of a genetic test for species membership unreliable. Using reproductive isolation as a definition of species will effectively deny the existence of a great deal of diversity that has traditionally been captured by descriptions of species.

Putting the matter another way, the Mayrian vision sees the cutting edge of evolution as isolated populations - incipient species - forging off into the future to find their unique destiny. A different view, made increasingly likely by the growing evidence of hybridization, proposes that many evolving groups will consist of a set of more or less hybridizing, though ecologically separated, kinds - but kinds sufficiently stable and robust to meet most traditional understandings of the species. Which of these pictures is correct is, at any rate, surely an empirical matter, and judging where and to what extent the latter situation obtains will again require the continuing engagement of taxonomists. And of course if it is not to be wholly question-begging, the relevant judgments will need to be based on a variety of criteria - morphological, behavioural, reproductive, etc. So the usefulness of genomic (barcode) taxonomy will be subject to the judgments of taxonomists, and the limiting factor on 'cataloguing life' will remain the availability of this expertise.

Costa and Carvalho also make the much more speculative suggestion that barcoding might greatly increase the interest in taxonomy among the general public, and thereby provide impetus for conservation measures. The basis for this suggestion is the vision of a hand-held barcoder - something that anyone could buy for $\$ 10$, according to one of the websites Costa and Carvalho reference for this proposal-connected by wireless link to a central databank. Though it is certainly easy to underestimate the rate of technical change in an area such as this, I am a little sceptical about this prediction. Still, let us assume for the sake of argument that such a thing is indeed forthcoming in a few years time. I remain sceptical as to whether such a product would find a mass market. As a (very) amateur taxonomist of wild plants, it is my experience that most people find the identification of flora and fauna decidedly uninteresting. And I suspect that those who do not, find the acquisition of the (currently) necessary skills a large part of the attraction of the practice. But more interestingly, and paralleling my point about professional taxonomy, it strikes me that the sort of knowledge people already interested in such matters have had to acquire would be necessary to make the use of the barcode reader rewarding. The great 
majority of plants, say, are, by definition, common. It is the expertise that enables one to pick out the uncommon or difficult-to-classify specimens that would make access to such a machine attractive. Constantly identifying brambles and stinging nettles would soon become tiresome.

I certainly don't wish to deny that the barcoding project has potential value to many kinds of users from professional taxonomists to enforcers of fishery protection legislation and amateur botanists and no doubt many others. It may even be a good investment of the very substantial resources it has attracted. But as with so many novel scientific projects nowadays, it has also attracted its fair share of hype. Suggestions that it will bring about the rapid cataloguing of all biodiversity, or that it will create a wave of popular excitement about taxonomy seem to me to belong in this category.

\section{Acknowledgements}

I am grateful to Maureen O'Malley for very helpful comments on an earlier draft of this paper. I gratefully acknowledge support from the Economic and Social Research Council (ESRC) and the Arts and Humanities Research Council (AHRC). The research in this paper was part of the programme of the ESRC Centre for Genomics in Society (Egenis).

\footnotetext{
${ }^{1}$ ESRC Centre for Genomics in Society (Egenis), University of Exeter, UK J.A.Dupre@exeter.ac.uk

${ }^{2}$ F.O. Costa and G.R. Carvalho. The Barcode of Life Initiative: synopsis and prospective societal impacts of DNA barcoding of fish. Genomics, Society and Policy 2007; 3 (2): 29-40.

${ }^{3}$ L. Van Valen. Ecological Species, Multispecies, and Oaks. Taxon 1976; 25: 233-239.

${ }^{4}$ P.R. Ehrlich and P.H. Raven. Differentiation of Populations. Science 1969; 165: 1228-1232.

${ }^{5}$ As a matter of fact success to date has been limited to animals. It has proved more difficult to find a suitable barcoding sequence for plants, and currently it appears that several sequences may be needed. See W.J. Kress and D.L. Erickson. A Two-Locus Global DNA Barcode for Land Plants: The Coding rbcL Gene Complements the Non-Coding trnH-psbA Spacer Region. PLoS ONE 2007; 2(6): e508. ${ }^{6}$ J. Mallet. Hybridization as an Invasion of the Genome. Trends in Ecology and Evolution 2005; 20: 229-237.
} 